\title{
Damage simulation for the disaster merit of light substances
}

\author{
P. Brož \\ Professional Association for Science, Research and Consultancy, \\ Czech Republic
}

\begin{abstract}
In general, dynamic failures of metal construction embrace a large variety of plastic strain rates. These influences must be taken via viscoplasticity, coupled with damage. For this problem two damage models, Gologanu's and Lemaitre's, are convenient; they are modified by the Hill potential to take account of material anisotropy. To stipulate the damage parameters of the model concerned, an inverse routine employing the optimizer to correlate the experimental and numerical responses of notched specimen tension tests is applied. This method was used to measure components being composed of the selected material and resulted in suitable values of damaged parameters in an appropriate time for numerical modelling. These quantities come from an optimization method that yields the best solution potential for a variety of possibilities. Both damage models by virtue of the damage mechanics are put on Gologanu's simulation, which concentrates on the progress of ellipsoidal microvoids in the course of plastic strain, and Lemaitre's, which applies a comprehensive damage variable that evolves the strain energy density release rate. When elaborating the damage parameter identification method, static and dynamic tests are utilized. The said damage system is used for the aluminium structural component.
\end{abstract}

Keywords: aluminium material, crash failure ductility, ellipsoidal microvoid, failure modelling, finite element codes, high strain rate, inverse technique.

\section{Introduction}

A great saving of weight, and disaster merit protection, is possible merely by the application of modern design intentions and use of lightweight substances, which have restricted ductility and a complicated failure. The failure possibility is thus 
a reality that could sharply vary the progression of deformation effects. The current crashworthiness codes are unsuitable to predict failure in such materials, or jointing methods that have raised serious doubts over their incomes. In order to prevent going back to a "prototype based design" and to maintain the high level of safety reached in recent years, it is an urgent necessity to better the failure prediction potentials of model systems of rules. This is why it has been urgent to take on a European research project called IMPACT [1], in order to evolve simulations and methodologies for failure prediction. The issues given involve metallic materials that suffer from complex failures under dynamic loadings. Thus, definite damage simulations based on the framework of continuum damage mechanics are implied to characterize the development of damage under mechanical loadings, its advance up to the initiation of a macrocrack and lastly the propagation of this macro-crack up to failure of the constituent. Gologanu's damage model, which describes all of these phenomena by introducing an accurate characterization of the evolution of microvoids under dynamic loadings, is applied [2]. The form and orientation of the microstructural cavities are considered in the finite element model developed to make better prediction of damage and fracture phenomenon, and furthermore the growth, nucleation and coalescence stages are taken into account to describe the evolution of the microvoid volume fraction, in their majority, according to [3]. The law of porosity evolution owing to nucleation is adapted to consider damage evolution in the course of pure shear loading.

A macroscopic way of tackling this problem, based on Lemaitre's model, is applied to symbolize pertinently the damage evolution subject to dynamic loading. Extended Lemaitre's simulation for ductile damage was set up to involve anisotropy in the damage development. The damage law is applied either in monotonic loadings for the ductile fracture or in cyclic loadings for low cycle or high cycle loadings. This law depends on the strain energy density release rate, which is the main variable for governing the damage event. The evolution damage law and threshold parameters have to be identified in the large plastic strain region when an inverse method is applied. Concurrently, the identification of the damage parameters lies in correlating an experimental and numerical macroscopic measurement. Dynamic tensile tests on notched specimens are used to measure macroscopic responses. The test data obtained is the change of the inner radius of the specimens in compliance with their elongation and also the change of forces according to the stated elongation. The damage models given simulate specimens and components for metal materials. Their capacity to predict the stress softening, damage and failure path is explained.

\section{Damage simulations}

A micro-mechanical approach using solid Finite Elements is applied to stipulate a quite exact prediction of the 3D stress classification in the contraction failure location and around a propagating crack. If suitably fine meshing and appropriate material laws are selected then both failure initiation and crack propagation can 
be represented; clearly this approach leads to large FE models and is thus restricted to constituent failure studies.

The term 'micro-mechanical models' is used to arrange a simulating approach using detailed 3D solid elements which attempt to represent local necking, the formation of a crack and crack growth.

\subsection{Gologanu damage model}

Gologanu model takes into account the alterations in microvoid form which occur during deformation. In fact, the Gologanu model considers cavities of ellipsoidal form, whose shape and orientation can evolve. The plastic potential is a quadratic formulation which can also be used as a yield function in which $\sigma_{\mathrm{eq}}$ is the macroscopic equivalent stress, $\sigma_{\mathrm{M}}$ is the elasto-viscoplastic flow stress, $q_{1} ; \alpha_{1}$ and $\alpha_{2}$ are material parameters introduced in order to converge the model with full numerical analyses of periodic arrays of voids; $f^{*}$ is the Tvergaard and Needleman's coalescence function:

$$
\phi_{\text {evp }}=C \frac{\left\|\sigma^{\prime}+\eta \sigma_{H} X\right\|^{2}}{\sigma_{M}^{2}}-\varphi=0
$$

where $\varphi=\mid 1+\left(q_{1} f^{*}\right)^{2}-2 q_{1} f^{*} \cosh (v), \quad v=\frac{\kappa \sigma_{H}}{\sigma_{M}}, \quad \sigma_{H}=\left(1-2 \alpha_{2}\right) \sigma_{11}+\alpha_{1} \sigma_{22}$

The parameters $\kappa, \eta, C$ and $X$ depend on the geometry of the ellipsoid void, $\sigma_{\mathrm{H}}$ is the mean stress and the von Mises norm can be written down as

$$
\left\|\sigma_{i j}\right\|=\sqrt{\frac{3}{2} \sigma_{i j} \sigma_{i j}}
$$

In pure shear loading, there is no damage evolution and consequently no rupture. In order to consider damage due to shearing the damage evolution law is modified to comprise of the sum of the classical law and a new part due to shear loading yielding,

$$
\dot{f}=\dot{f}_{\text {growth }}+\dot{f}_{\text {nucleation }}+\dot{f}_{\text {shear }}
$$

In pure shear it is commonly accepted that the voids experience a rotation without any change in growth and it appears that nucleation can be generated. Consequently, the damage evolution law due to shearing takes the form of a statistical law, similar to the nucleation evolution law, but taking into consideration the shearing strain and the shearing strain rate. It is given by,

$$
\dot{f}_{\text {shear }}=\frac{f_{s}}{S_{s} \sqrt{2 \pi}} \exp \left(-\frac{1}{2}\left(\frac{\varepsilon_{x y}-\varepsilon_{s}}{S_{S}}\right)^{2} \dot{\varepsilon}_{x y}\right)
$$



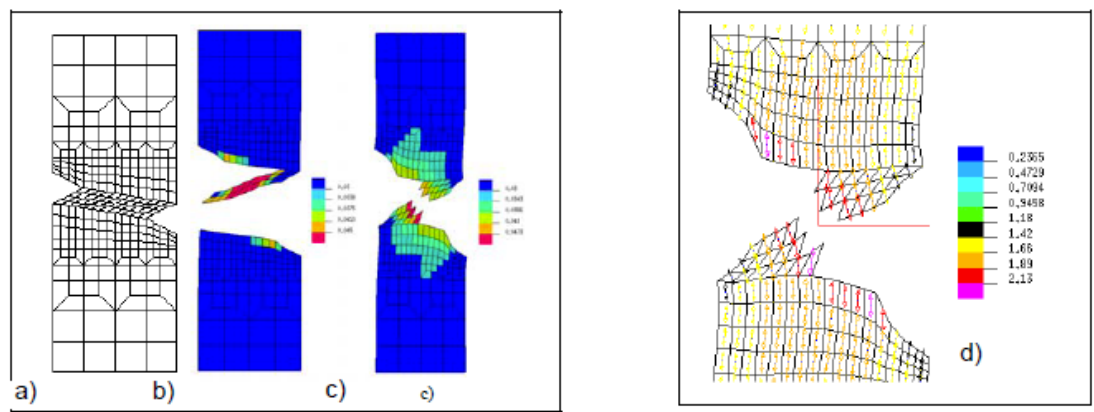

Figure 1: Comparison of rupture mechanisms for Gurson and Gologanu models (taken from [3]).

The identification of the damage parameters controlled by the shearing strains is carried out by the inverse method using an Arcan type test. Fig. 1 shows the tensile simulation of a non-axisymmetric double V-notched specimen; a) shows the initial finite element modelling; b) and c) are the damage distribution at the end of the process using Gologanu model. Fig. 1d gives the orientation and shape evolution $\mathrm{S}$ at the end of the rupture process for an initial prolate void shape using Gologanu model. From this figure it can be seen that including changes in the void shape does allow anisotropic damage to be represented and more correctly captures the failure process. It should be noted, that this implementation was only for shell element which restricts precision as triaxial stresses, especially in the necking zone, are ignored. A solid element would end in better results but would be CPU time consuming; an additional progression could be to apply an energy criterion to dissipate energy because each failed element is got rid of.

\subsection{Lemaitre damage model}

The framework of continuum damage mechanics is used to describe the development of damage under mechanical loadings, its progression up to the initiation of a macro-crack and finally the growth of this macro-crack during failure of the component. The original model for ductile fracture was established for isotropic damage conditions and later extended to include anisotropic damage development $[11,12]$. The basic ingredient of the model is the damage law used either in monotonic loadings for ductile fracture, or in cyclic loadings for low cycle or high cycle loadings. This damage law depends on the damage variable $D$ and the strain energy density release rate which is the principal variable governing the phenomenon of damage and is expressed by,

$$
Y=\frac{W_{e}}{1-D}
$$

The strain energy rate $W e$ is split into its shear and hydrostatic parts, and leads to the following expression, 


$$
W_{e}=\frac{(1+v)}{2 E(1-D)}\left\langle\sigma_{i j}\right\rangle\left\langle\sigma_{i j}\right\rangle-\frac{v}{2 E(1-D)}\left\langle\sigma_{i j}\right\rangle^{2}
$$

in which $\left\langle\sigma_{i j}\right\rangle=\sigma_{i j}$ if $\sigma_{i j}>0$ and $\left\langle\sigma_{i j}\right\rangle=0$ if $\sigma_{i j}<0$. Lastly, the damage evolution during plastic straining is formulated by the expression as follows,

$$
\dot{D}=\left(\frac{Y}{S}\right)^{S} \dot{\varepsilon}^{p} \quad \text { if } \quad \varepsilon^{p}>\varepsilon_{D}
$$

where $S$ and $s$ are material coefficients, $\varepsilon^{p}$ is the effective plastic strain and $\varepsilon_{D}$ is the plastic strain at damage threshold.

Since the damage evolution is localized in the large plastic strain zone, the evolution damage law and the threshold parameters have to be consequently identified. A direct identification approach or local approach is used essentially on uniaxial monotonic and cyclic tests, Fig. 2. For a better identification experiments in the largest possible domains of stresses, strains, time and number of cycles are needed. An identification approach using inverse techniques is used to find the damage parameters by correlating experimental and numerical macroscopic measurement strongly dependent on the parameters. Tensile tests on thin notched specimens are used as mechanical tests to measure macroscopic responses and variations of inner radius and force with respect to elongation of the specimen employed to correlate experiment and numerical simulations.

In order to better the characteristics of an aluminium alloy, an anisotropic potential can be applied; in the stated case, a Hill 48 potential is considered. The above damage model for anisotropic materials was implemented in 2D shell and 3D solid elements. The damage parameters were identified as treated above and applied to a validation example consisting of the three points bending of an

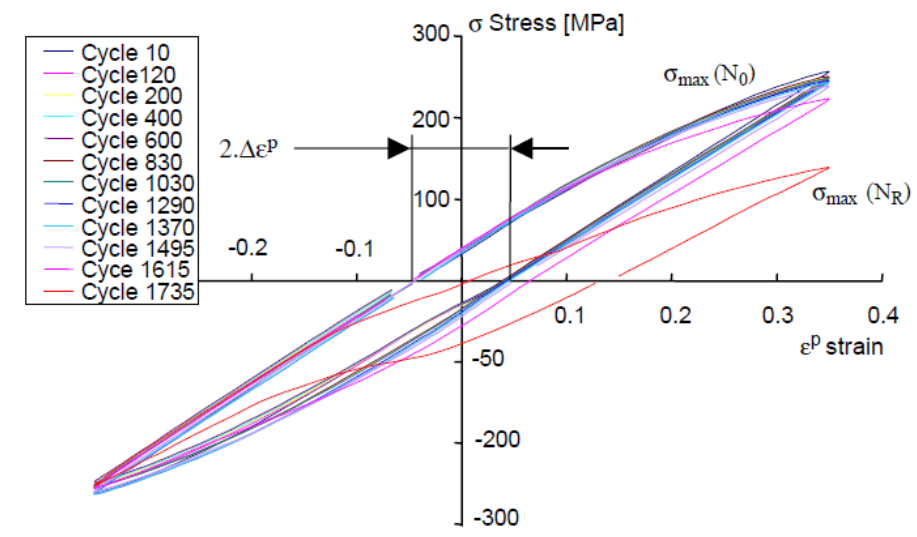

Figure 2: Monotonic cyclic test to determine Lemaitre damage parameters. 


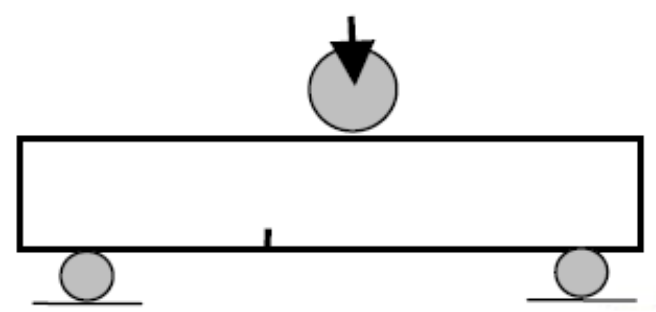

Figure 3: $\quad$ FE model for the 3 point bending of an aluminium section with 'starter crack'.

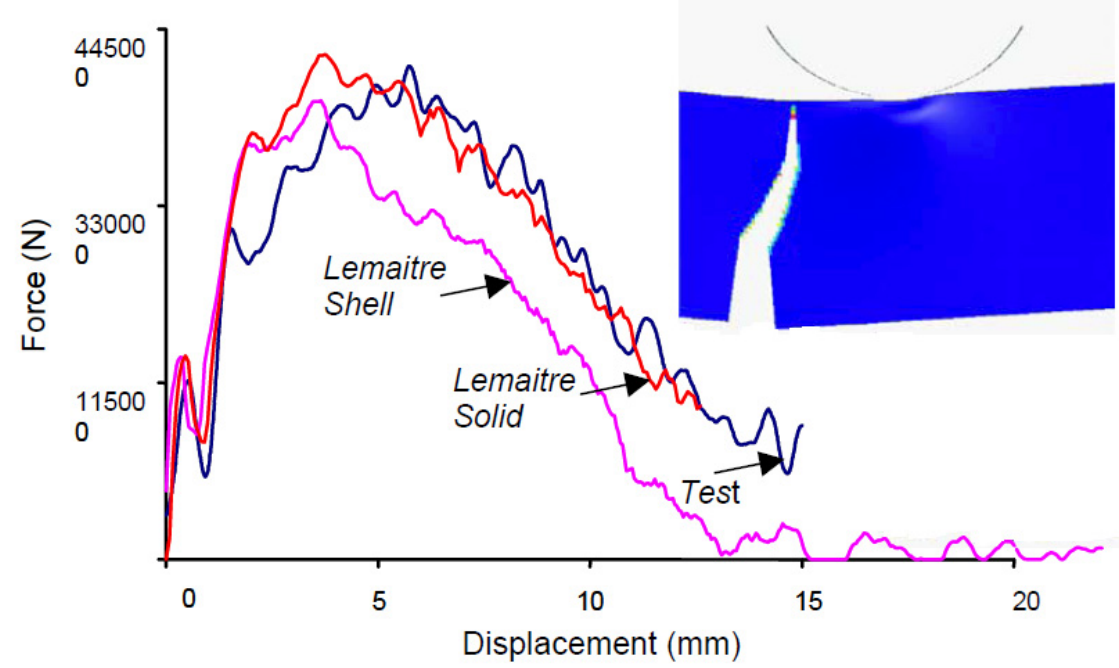

Figure 4: Experimental and simulation load versus displacement curves for shells and solids (Lemaitre model).

aluminium extruded section with an initial narrow opening (initial crack) on the lower face to localize failure initiation, Fig. 3. This test was performed dynamically and the numerical and experimental results are found to be in good correspondence as regards failure path and energy rank, Fig. 4.

\section{Damage parameters identification}

Founded on an inverse technique, an identification method is used. This procedure lies in the identification of the damage parameters by correlating, with an optimization process, an experimental and numerical macroscopic measurement considerably dependent on the parameters $[4,5]$. In tensile tests, the notched flat specimens 2, 3 and $4 \mathrm{~mm}$ radii are applied. The variation of both in the bottom of the notch and the variation of the axial force in function of the 
elongation of the notched specimen are employed as macroscopic measurements. The finite element simulations of the tests are performed with the exact boundary conditions of the experimental set up. For strain rate sensitive materials like steel, the tensile tests are performed for various plastic strain rates, covering the range of crash tests $\left(0\right.$ to $\left.500 \mathrm{~s}^{-1}\right)$ by employing a quasi-static test machine, servohydraulic device and Hopkinson bar [6]. A high speed camera, laser extensometer and representation analyzer are used. In the case of dynamic loading, to measure the macroscopic response, an imaging correlation program was evolved to obtain the experimental information. Next, the measurements before defined are calculated following the evolution of the form of the specimen by dint of a speed camera and home-made software. For Hopkinson bars merely the strength against elongation of the specimen is employed owing to the pure number of images gained at high speed loadings.

Lastly, the correlation is obtained by minimizing a cost function that is explained by the least square approximation being:

$$
Q(\alpha)=\sum_{i=1}^{n b} \frac{\left[Z_{i}^{\text {sim }}(\alpha)-Z_{i}^{\text {exp }}\right]^{2}}{\left[Z_{i}^{\exp }\right]^{2}}
$$

where $\alpha$ are material parameters to identify, $Z^{\text {sim }}$ and $Z^{\text {exp }}$ are simulated and experimental macroscopic responses $n b \_$point is the number of experimental points of the response.

The scalar material parameters are $\alpha=\left\{q_{1}, f, f_{N}, S_{N}, \varepsilon_{N}, S, f_{c}, f_{F}\right\}$ and $\alpha=\left\{\varepsilon_{D}\right.$, $\left.D_{c}, S, s\right\}$ in the case of Gologanu's model and Lemaitre's model. A first initial curve named "numerical non optimized" in Fig. 5 is obtained with the initial parameters and following some iterations comes to be the "numerical optimized" using the optimized parameters.

The damage parameters of $6014 \mathrm{~T} 7$ aluminium alloy are recognized using the identification routine indicated (Table 1). This aluminium material has a high ductility with a weak anisotropy and damage owing to the extrusion process. As a consequence, the critical damage $D_{c}$ is low for Lemaitre's model and the critical microvoid volume fraction at coalescence beginning $f_{c}$ is low, too, in view of the initial damage $f_{0}$ for Gologanu's model - for this simulation the nucleation belongs especially to a very low equivalent plastic strain that fast increases the damage value.

Table 1: $\quad$ Damage parameters of $6014 \mathrm{~T} 7$ aluminium alloy.

\begin{tabular}{|l|cccccccc|}
\hline Gologanu's & $\mathrm{q}_{1}(-)$ & $\mathrm{S}_{0}(-)$ & $\mathrm{f}_{N}(-)$ & $\mathrm{S}_{N}(-)$ & $\varepsilon_{N}(-)$ & $\mathrm{f}_{c}(-)$ & $\mathrm{f}_{F}(-)$ & $\mathrm{f}_{0}(-)$ \\
\cline { 2 - 8 } model & 1.52 & 0.001 & 0.040 & 0.1 & 0.19 & 0.06 & 0.08 & 0.001 \\
\hline \multirow{2}{*}{$\begin{array}{l}\text { Lemaitre's } \\
\text { model }\end{array}$} & $\varepsilon_{D}(-)$ & $\mathrm{s}(-)$ & $\mathrm{S}(\mathrm{MPa})$ & $\mathrm{D}_{c}(-)$ & & & & \\
\cline { 2 - 9 } & 0.05 & 2 & 1.22 & 0.34 & & & & \\
\hline
\end{tabular}




\section{Extruded pipe}

A notched extruded pipe was dynamically tested on an impact bending at 3 meters $/$ second with $50 \mathrm{~mm}$ radius impactor of $275 \mathrm{~kg}$ weight. The model consists of 15614 shell elements. For the solid model, the mesh comprises three elements over the thickness. The failure is modelled by element elimination for both considered damage models.

For the $6014 \mathrm{~T} 7$ which is an orthotropic substance, material parameters are identified using $0^{\circ}, 45^{\circ}$ and $90^{\circ}$ tensile experiments from the rolling direction (Table 2). The law of characteristics in $0^{\circ}$ direction is indicated in Fig. 5. Experimental and numerical results are in good conformity as regards the failure moment, failure path and energy dissipation primarily in the case of Lemaitre's model. Gologanu's model using shell elements is exact till the first element elimination adding after that, the strain control of damage results in an underestimation of the energy dissipation along the failure. For all that, when using shell element, finite element models lead to an underestimation of the dissipated energy which is defined by the fact that shell elements cannot consider the through thickness stresses even for seven integration points through the thickness. In the case of solid elements, the outcomes are extra auspicious.

\section{Effect of stress triaxility and strain rate on the characteristics of a constructive material}

Constitutive relations for metallic materials at high strain rates change from empirical to theoretical relationships, based on the micro-mechanical processes

Table 2: HILL 48 material parameters.

\begin{tabular}{ccccc}
\hline $\mathrm{F}$ & $\mathrm{G}$ & $\mathrm{L}$ & $\mathrm{M}$ & $\mathrm{N}$ \\
\hline 1.049 & 1.3 & 3 & 3 & 3.13 \\
\hline
\end{tabular}

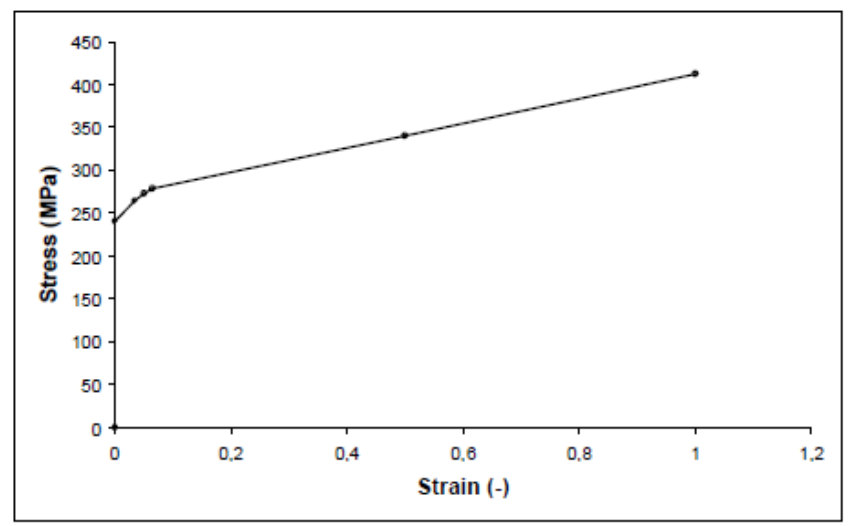

Figure 5: Behaviour law in the $0^{\circ}$ direction for $6014 \mathrm{~T} 7$ aluminium alloy [3]. 
supposed to determine the macroscopic characteristics of the material. Normally, the constitutive relation defines the equivalent stress $\sigma$ in terms of equivalent (or accumulated) plastic strain $\varepsilon_{p}$, equivalent plastic strain rate $\varepsilon_{p}$ and temperature $T$. In principle, the material parameters in the constitutive equations are identified founded on test data brought from tension or torsion tests for considerable extends of strain rate and temperature.

Although the strength of metallic materials depends on strain, strain rate and temperature, much experimental checking indicates that the ductility depends substantially on the triaxiality of the stress state. The stress triaxiality is characterized by the dimensionless parameter $\sigma_{m} / \sigma$, where $\sigma_{m}$ stands for the mean stress. Computational fracture simulations were presented that include the effect of stress triaxility and the influences of strain rate and temperature. The influence of the stress triaxility in these models is based on the void growth equation. It is familiar to fit the material parameters of the computational fracture models to date obtained from tensile tests with notched axisymmetric specimens. Various stress triaxility levels are obtained by changing the notch radius of the specimens.

The goal is to investigate the effect of stress triaxility and strain rate on the characteristics of a structural steel and to get experimental data that may be used to legalize constitutive relations and fracture criteria. At last, notched axisymmetric specimens of S 460 steel with three different notch radii were tested in tension at two different strain rates. The tests were carried out in a Split Hopkinson Tension Bar, using a digital high-speed camera system to record the local deformation in the notch. Next, computer models are performed to validate the constitutive relation and fracture model, and to detailed analysis of the material characteristics in the course of high-rate tension tests of smooth and notched samples.

\subsection{Experimental program}

The specimens were made from a $12 \mathrm{~mm}$ thick plate of $460 \mathrm{E}$ steel that is a thermo - mechanically rolled ferritic fine grained structural steel proposed high strength combined with a high degree of ductility. All specimens were taken parallel to the rolling direction of the steel plate. A slight normal anisotropy was achieved.

The geometry and dimensions of the notched specimens are given in Fig. 6. Tests were carried out for three different notch root radii $R_{0}$ equal to $0.4 \mathrm{~mm}, 0.8$ $\mathrm{mm}$ and $2 \mathrm{~mm}$. Two levels of loading rate were applied, determined by a preloading force $N_{0}$ of the modified Split Hopkinson bar apparatus equal to $20 \mathrm{kN}$ and $40 \mathrm{kN}$. As a result, the total number of tests was 25 . A test identification system is chosen here where, e.g. R0.8 - 20-4 has the following meaning: R0.8 - notch radius $R_{0}=0.8 \mathrm{~mm} ; 20$ - pre-loading force $N_{0}=20 \mathrm{kN} ; 4$ - parallel test No. 4.

The tensile tests of the notched specimens were carried out in a Split Hopkinson Tension Bar at the European Commission Joint Research Centre in Ispra, Italy. A drawing of the Split Hopkinson Tension Bar is demonstrated in Fig. 7. 


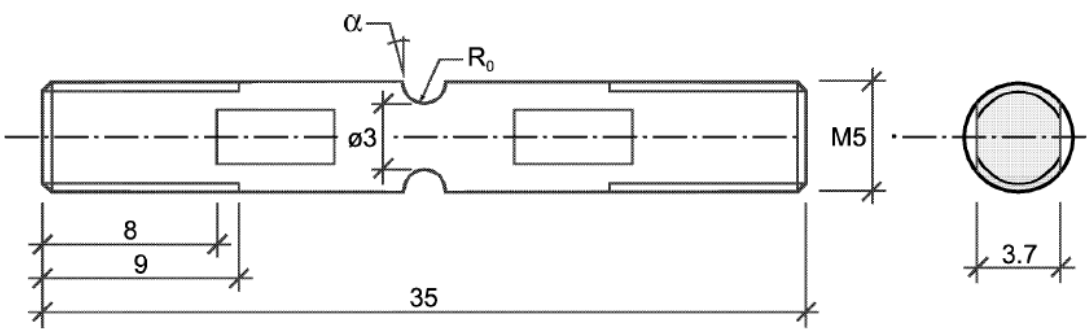

Figure 6: Geometry and dimensions (in $\mathrm{mm}$ ) of notched test specimens for high rate experiments in the Split Hopkinson Tension Bar (taken from [6]).

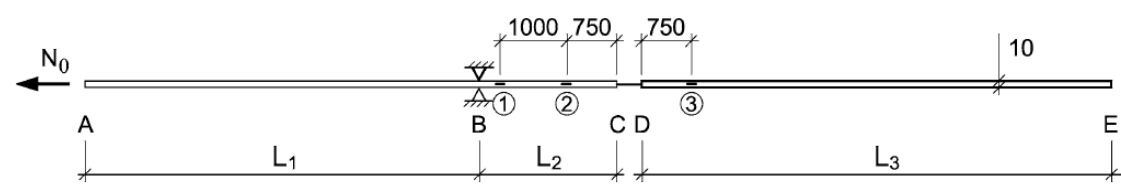

Figure 7: Sketch of the Split Hopkinson Tension Bar (in mm), where $L_{1}=$ $5870 \mathrm{~mm}, L_{2}=2055 \mathrm{~mm}$ and $L_{3}=5800 \mathrm{~mm}$. The diameter of the input bar $\mathrm{A}-\mathrm{B}-\mathrm{C}$ and the output bar $\mathrm{D}-\mathrm{E}$ is $10 \mathrm{~mm}$.

The test apparatus consists of two half - bars named the input bar A - B - C and the output bar D-E, and the specimen $\mathrm{C}-\mathrm{D}$ is inserted between these two bars. The pre-loading force $N_{0}$ is applied to the left further point of the input bar A, while the bar is fixed in the longitudinal direction with a brittle intermediate portion at $\mathrm{B}$. By rupturing the brittle intermediate piece be, a tension wave with a rise-time of about $50 \mu \mathrm{s}$ is transmitted along the input bar and load the specimen to fracture. The strain rate is varied by changing the pre-loading force $N_{0}$ of the loading device and so the stored elastic energy.

According to the theory of one-dimensional elastic wave propagation in circular rods, the elastic stress wave propagates along the input bar with a constant velocity and form. When the incident pulse achieves the specimen, it is partly reflected at the interface between the input bar and the specimen, and partly transmitted throughout the specimen and into the output bar. The relative amplitudes of the event, reflected and transmitted pulses depend on the mechanical properties of the specimen. In is supposed that the specimen, which is short relative to the input and output bars, reaches a force equilibrium state after several reflections of elasto-plastic waves in it.

\section{Conclusion}

Gologanu and Lemaitre damage models were adapted by bringing in Hill potential to consider anisotropy of the material. An inverse routine applying to correlate experimental and numerical responses of notched specimen tensile tests is used to stipulate the damage parameters of both damage simulations. This 
technique was applied to an aluminium component and resulted in relevant values of damage parameters in a suitable time for numerical modelling. These values originate from an optimization method which yields the best solution.

An impact bending test on a notched aluminium extruded tube to locate the failure was carried out. Generally, the predicted energy dissipation and the failure moment and growth were determined to be in good harmony with the experimental steps. Anyhow, because both identification techniques are mesh dependent, a regularization procedure should be applied to prevent this dependency. Both damage models can be employed for crash simulations. Gologanu damage simulation is very precious and the use of ellipsoidal microvoids permits to possess a damage anisotropic evolution. Indeed, the complexity of the model is a liability for industrial applications. Lemaitre damage model is easier to employ and identify owing to the small number of parameters. The stress check of the damage evolution is definitely an asset in the simulation.

\section{Acknowledgement}

The author gratefully acknowledges the financial support of the presented research by the Grant Agency of the Czech Republic (project No. 103/09/1580).

\section{References}

[1] Framework $\mathrm{V}$ project. Improved failure prediction for advanced crashworthiness of transportation vehicles. G3RD-CT-2000-00186, 20002003.

[2] Gologanu, M., Leblond, J.B. \& Devaux, J., Numerical and theoretical study of coalescence of cavities in periodically voided solids. Computational Material Modelling, 42, pp. 223-244, 1994.

[3] Bennani, B. \& Lauro, F., damage models and identification procedures for crashworthiness of automotive light materials. Latin American Journal of Solids and Structures, 3, pp. 75-87, 2006.

[4] Lauro, F., Bennani, B., Croix, P. \& Oudin, J., Identification of the damage parameters for anisotropic materials by inverse technique: application to an aluminium. Journal of Materials Processing Technology, 118, pp. 472-477, 2001.

[5] Brož, P. \& Dobiáš, D., Determining material parameters of damage. Acta Technica, AS CR, Prague, 54(4), pp. 415-436, 2009.

[6] Hopperstad, O.S., Børvik, T., Langseth, M., Labibes, K. \& Albertini, C., On the influence of stress triaxiality and strain rate on the behaviour of a structural steel. Part I. Experiments, European Journal of Mechanics A/Solids, 22, pp. 1-13, 2003. 